\title{
The association of absolute eosinophil count, serum immunoglobulin E and spirometry with co-morbid bronchial asthma in patients with allergic rhinitis
}

\section{Neelima Vijayan', Padmanabhan Karthikeyan', Nirmal Coumare Venkataramanujam ${ }^{\text {, }}$ Ramiya Ramachandran Kaipuzha², Davis Thomas Pulimoottil ${ }^{3}$}

${ }^{1}$ Department of Otorhinolaryngology, Mahatma Gandhi Medical College and Research Institute, Sri Balaji Vidyapeeth University, Pondicherry, India

${ }^{2}$ Department of Otorhinolaryngology and Head and Neck Surgery, Jahra Hospital, Al Jahra, Kuwait

${ }^{3} \mathrm{Al}$ Azhar Medical College and Super Specialty Hospital, Ezhalloor, Thodupuzha, Kerala, India

\section{ABSTRACT}

OBJECTIVE. This study aimed to analyse the association of absolute eosinophil count (AEC), serum IgE and spirometry with co-morbid bronchial asthma in patients with allergic rhinitis.

MATERIAL AND METHODS. This study involved 50 patients with signs and symptoms of allergic rhinitis who underwent a clinical examination and various tests, including spirometry, and were followed up regularly. Patients found to have bronchial asthma or nasal polyposis were treated accordingly.

RESULTS. The study found the prevalence of bronchial asthma in patients with allergic rhinitis to be $58 \%$ and that the severity of bronchial asthma was reduced significantly, with lesser acute attacks and reduced hospitalizations with the effective treatment of allergic rhinitis ( $\mathrm{p}=0.064)$. CONCLUSION. This study showed that elevated AEC and serum IgE were significantly associated with co-existing allergic rhinitis and bronchial asthma and increased the chance of co-existence of these two pathologies. Spirometry is a useful tool for observing the response to treatment. KEYWORDS: allergic rhinitis, bronchial asthma, comorbidity, spirometry, nasal polyps.

\section{INTRODUCTION}

Allergic Rhinitis is a symptomatic condition which causes significant social, functional impairment and decreased work performance and productivity $^{1}$. Bronchial asthma is another chronic disease which affects a patient socially, physically and emotionally. The probability of development of asthma is much higher in individuals with both perennial and seasonal rhinitis than in individuals with either condition alone ${ }^{2}$. Asthma and rhinitis were found to be co-morbid conditions, regardless of the atopic state, and raised bronchial hyperresponsiveness was seen in patients with rhinitis ${ }^{3}$. The above two conditions may be manifestations of an inflammatory process within a continuous airway, rather than two separate diseases, hence labelled as 'One Airway, One Disease' or 'United Airways Disease'.

\section{MATERIAL AND METHODS}

After receiving the approval of the Institutional Ethical Committee, 50 subjects who had presented to the Outpatient Department of the ENT Department of a rural tertiary care centre with symptoms and signs of allergic rhinitis were enrolled in this study. The exclusion criteria included: acute or chronic sinusitis, tuberculosis, cystic fibrosis, chronic obstructive pulmonary disease and smokers. After signing the written and informed consent, the subjects underwent a complete blood count, absolute eosinophil count, serum IgE, which were then followed by radiological tests that included X-ray of the nose and paranasal sinuses (Water's view) and computed tomography of the nose and paranasal sinuses (in those cases who had nasal polyposis). Examination of the stools for ova and cysts to rule out helminth para- 


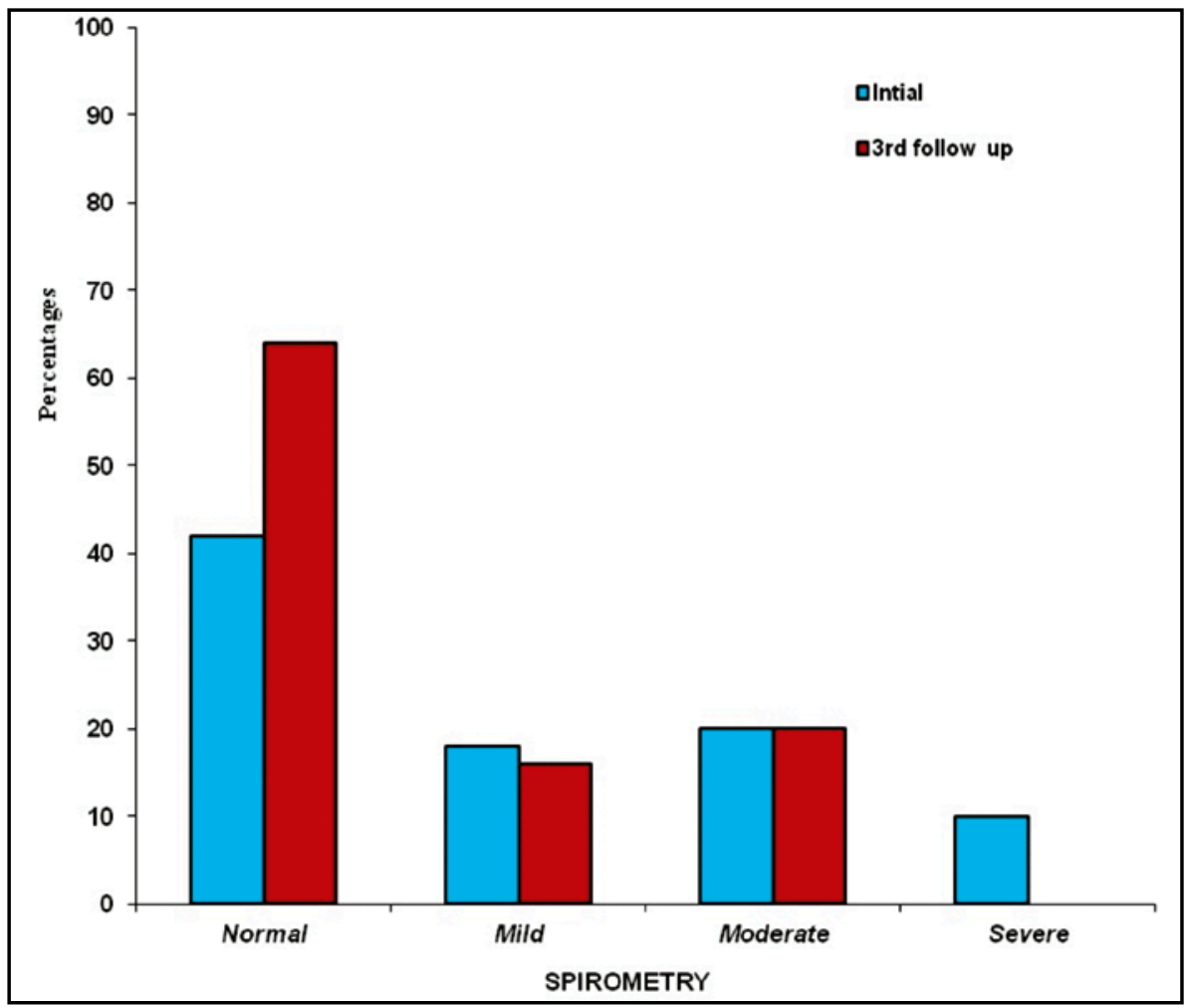

Figure 1 Comparison of spirometry findings in patients at the initial visit and at the third follow-up.

sitic infestation contributing to allergic manifestations and sputum sampling for acid-fast bacilli were done for all 50 subjects. The patients were then subjected to diagnostic nasal endoscopy, after which they were evaluated by a Pulmonologist for the presence of asthma. A spirometric evaluation was done and the subjects were subsequently categorized into those with and without bronchial asthma, after which the appropriate treatment was started.

All 50 subjects were treated medically and the 12 subjects who had sinonasal polyposis were treated surgically by a functional endoscopic sinus surgery followed by medical treatment. All 50 subjects were given a steroid nasal spray - fluticasone furoate nasal spray - after the 2-3-week treatment with oral leukotriene receptor antagonist and antihistamines. This spray was given for 6 months and at the end of those 6 months, the spirometry was repeated.

\section{RESULTS}

In our study, out of the 50 subjects, the prevalence of allergic rhinitis with bronchial asthma was found to be $58 \%$ (29 subjects) and the prevalence of subjects with only allergic rhinitis without coexisting asthma was found to be $42 \%$. Out of the 29 subjects who were found to have asthma, 18
$(62.07 \%)$ were females and $11(37.93 \%)$ males. All of the 50 subjects had normal white blood cell counts, thus ruling out sinusitis and other infective conditions. 42 subjects $(84 \%)$ had raised absolute eosinophil count (AEC) and $8(16 \%)$ were found to have normal counts. All of the 29 patients who had asthma with rhinitis were found to have raised counts. Serum IgE was elevated in 40 subjects $(80 \%)$ and all asthmatic subjects had elevated levels of IgE.

The plain X-ray of the nose and paranasal sinuses showed abnormality in 41 subjects $(82 \%)$, which included turbinate hypertrophy and mucosal thickening of the sinuses. Nasal discharge for culture and sensitivity sent for all 50 subjects were reported to be sterile, thus supporting the diagnosis of allergic rhinitis.

In the initial spirometry done at the beginning of study before starting the treatment, it was found that 21 patients $(42 \%)$ had a normal test, while 29 patients $(58 \%)$ showed obstruction with reversibility, which indicates the presence of bronchial asthma. Out of the 29 subjects, $9(31.03 \%)$ had mild obstruction with reversibility, $10(34.48 \%)$ had moderate obstruction with reversibility and 10 $(34.48 \%)$ had severe obstruction with reversibility.

Three follow-ups were carried out in the $1^{\text {st }}, 3^{\text {rd }}$ and $6^{\text {th }}$ months after starting the treatment, and all 


\section{Table 1}

Comparison of patient characteristics with spirometric findings and corresponding Odds ratio

\begin{tabular}{|c|c|c|c|c|c|c|c|}
\hline \multirow{2}{*}{\multicolumn{2}{|c|}{ Characteristic }} & \multicolumn{2}{|c|}{ Spirometry findings } & \multirow{2}{*}{$\begin{array}{l}\text { Total } \\
\text { n (\%) }\end{array}$} & \multirow[b]{2}{*}{$x^{2}$} & \multirow[b]{2}{*}{$p$-value } & \multirow[b]{2}{*}{ Odds ratio } \\
\hline & & $\begin{array}{c}\text { Abnormal } \\
\mathbf{n}(\%)\end{array}$ & $\begin{array}{c}\text { Normal } \\
\text { n (\%) }\end{array}$ & & & & \\
\hline \multirow{2}{*}{ AEC } & Elevated & $29(69.05)$ & $13(30.95)$ & $42(84)$ & \multirow{2}{*}{8.9534} & \multirow{2}{*}{0.0028} & \multirow{2}{*}{15.6} \\
\hline & Normal & $1(12.5)$ & $7(87.5)$ & $8(16)$ & & & \\
\hline \multirow{2}{*}{ Serum lgE } & Elevated & $29(72.5)$ & $11(27.5)$ & $40(80)$ & \multirow{2}{*}{9.3591} & \multirow{2}{*}{0.0022} & \multirow{2}{*}{10.6} \\
\hline & Normal & $2(20)$ & $8(80)$ & $10(20)$ & & & \\
\hline \multirow{2}{*}{ Plain X-ray PNS } & Abnormal & $21(51.22)$ & $20(48.78)$ & $41(82)$ & \multirow{2}{*}{4.2989} & \multirow{2}{*}{0.0381} & \multirow{2}{*}{0.13} \\
\hline & Normal & $8(88.89)$ & $1(11.11)$ & $9(18)$ & & & \\
\hline
\end{tabular}

subjects were found to be symptomatically better at the end of 6 months.

The spirometry performed at the $3^{\text {rd }}$ follow-up, showed a significant step down in all of the 29 subjects. $9(31.03 \%)$, who initially had mild obstruction with reversibility, were found to have normal results in the repeated study. Out of the 10 $(34.48 \%)$ who had moderate obstruction with reversibility, 2 had normal results in the repeated test and 8 had stepped down to mild obstruction with reversibility. Out of the $10(34.48 \%)$ subjects who had severe obstruction, it was seen that all 10 had stepped down to moderate obstruction with reversibility. The improvement was significant, with a p-value of 0.064 (Figure 1).

The statistical analysis of the patients' variables showed a significant association between elevated AEC and serum IgE and abnormal findings on plain X-ray of the nose and paranasal sinuses and allergic rhinitis with bronchial asthma. Based on the Odds ratio, patients with allergic rhinitis with elevated AEC and elevated serum IgE were 15 times and 10 times, respectively, more at risk to be associated with co-existing bronchial asthma (Table 1$)$.

\section{DISCUSSIONS}

Epidemiologic studies have suggested that asthma and allergic rhinitis often coexist in the same patient. In our study, the prevalence of bronchial asthma in allergic rhinitis patients was found to be $58 \%$. A study done by A Navarro et al. showed a high prevalence of bronchial asthma in allergic rhinitis patients which can affect as many as $89.5 \%{ }^{2}$. In our study, serum IgE levels were estimated for all subjects and it was found that $40(80 \%)$ subjects had elevated levels and $10(20 \%)$ had normal levels. S Manohar et al. showed that estimation of serum $\operatorname{IgE}$ is a dependable laboratory data in patients suffering from allergic rhinitis and asthma ${ }^{4}$, while Agam Vora et al. states that high serum levels of allergen-specific IgE that are directed towards aeroallergens or environment characterize allergic diseases like rhinitis and asthma ${ }^{5}$.

A plain X-ray of the paranasal sinuses was taken for all 50 subjects included in the present study and $9(18 \%)$ were found to be normal and 41 $(82 \%)$ were found to have different abnormalities, like inferior turbinate hypertrophy $(13,31.70 \%)$, mucosal thickening $(19,46.34 \%)$ and a combination of both $(9,21.95 \%)$. Our findings were in accordance with EO Meltzer who stated that $40-60 \%$ of patients with asthma have abnormal X-rays of the paranasal sinuses ${ }^{6}$.

Our subjects underwent spirometry and, out of them, $29(58 \%)$ subjects had an obstructive reversible pattern suggestive of bronchial asthma. In a study performed by Navarro et al., 742 (79\%) of 968 patients underwent spirometry and it was found to be normal in $90 \%$ of the patients; the authors also observed no significant difference in spirometric parameters between patients with asthma or with both asthma and rhinitis ${ }^{2}$.

$12(24 \%)$ of the subjects evaluated by us were found to have sinonasal polyposis along with bronchial asthma. These subjects underwent endoscopic polypectomy followed by treatment for allergy and an improvement in asthma symptoms was seen in these subjects. Senior et al. states that asthma symptoms are reduced in those patients in whom functional endoscopic sinus surgery has been performed ${ }^{7}$.

In our study, all 50 subjects were given a steroid nasal spray - fluticasone furoate nasal spray - after 
the 2-3-week treatment with oral leukotriene receptor antagonist and antihistamines. This spray was given for 6 months and, at the end of these 6 months, all subjects were symptomatically better. Pedro Giavina-Bianchi et al. states that fluticasone furoate nasal spray "has a high topical potency and low potential for systemic effects" and is proved to be a good treatment for rhinitis ${ }^{8}$, which is in accordance with our findings.

\section{CONCLUSIONS}

The co-existence between allergic rhinitis and bronchial asthma is very strongly supported by genetic, pathophysiologic and clinical evidence. The treatment for allergic rhinitis in these patients alleviates the symptoms of co-morbid bronchial asthma. An elevated AEC and serum IgE along with an abnormal radiograph of the paranasal sinuses should raise a suspicion of the possibility of co-existing bronchial asthma in the clinicians' mind. This study supports the concept that both allergic rhinitis and bronchial asthma are a revelation of one disease entity and that the severity of asthma reduces considerably with the treatment of allergic rhinitis, with lesser acute attacks, reduced hospitalizations and a better quality of life.

Acknowledgement. The authors would like to thank Dr. G. Ezhumalai, Senior Statistician, Sri Balaji Vidyapeeth University, Pondicherry, for the immense help proffered for the statistical analysis for this study. The authors are also grateful to the authors of all those articles from where the literature for this article has been reviewed and discussed.

Conflict of interest. The authors have no conflict of interest.

Ethical standards. The authors assert that all procedures contributing to this work comply with the ethical standards of the Institutional Research Committee and with the Helsinki Declaration of 1964, as revised in 2008. All patients gave their informed consent for their inclusion in the study. This study does not contain any studies with animals performed by any of the authors.

\section{REFERENCES}

1. Hansel FK. Clinical and histopathologic studies of the nose and sinuses in allergy. I Allergy. 1929;1(1):43-70.

2. Navarro A, Valero A, Julia B, Quirce S. Coexistence of asthma and allergic rhinitis in adult patients attending allergy clinics: ONEAIR study. J Investig Allergol Clin Immunol. 2008;18(4):233-8.

3. Coca AF, Cooke RA. On the classification of the phenomena of hypersensitiveness. J Immunol. 1923;8(3):163-82.

4. Manohar S, Selvakumaran R. Estimation of serum immunoglobulin E (IgE) level in allergic asthma and allergic rhinitis patients before and after treatment. Eur J Exp Bio. 2012;2(6):2199-205.

5. Vora A. Anti IGE therapy in allergic asthma and allergic rhinitis. Medicine Update. 2012;22:370-7.

6. Meltzer EO, Szwarcberg J, Pill MW. Allergic rhinitis, asthma, and rhinosinusitis: diseases of the integrated airway. J Manag Care Pharm. 2004;10(4):310-7.

7. Senior BA, Kennedy DW, Tanabodee J, Kroger H, Hassab M, Lanza DC. Long-term impact of functional endoscopic sinus surgery on asthma. Otolaryngol Head Neck Surg. 1999;121(1):66-8.

8. Giavina-Bianchi P, Agondi R, Stelmach R, Cukier A, Kalil J. Fluticasone furoate nasal spray in the treatment of allergic rhinitis. Ther Clin Risk Manag. 2008;4(2):465-72. 